\title{
Pilot Tone Design for Dispersion Estimation in Coherent Optical Fast OFDM Systems
}

\author{
J. Zhao ${ }^{1}$ and A.D. Ellis ${ }^{1,2}$ \\ ${ }^{I}$ Photonic Systems Group, Tyndall National Institute and Department of Physics, University College Cork, Lee \\ Maltings, Prospect Row, Cork, Ireland, \\ ${ }^{2}$ now with Aston Institute of Photonic Technology, Aston University, Birmingham B4 7ET, UK \\ Tel.: 353 (21) 490 4128; Email: jian.zhao@tyndall.ie
}

\begin{abstract}
:
We show that inserting pilot tones with frequency intervals inversely proportional to the subcarrier index exhibits greatly improved dispersion estimation performance when compared to the equal spacing design in optical fast orthogonal frequency division multiplexing (F-OFDM). With the proposed design, a 20-Gbit/s four amplitude shift keying optical F-OFDM system with $840-\mathrm{km}$ transmission without optical dispersion compensation is experimentally demonstrated. It is shown that a single F-OFDM symbol with six pilot tones can achieve near-optimal estimation performance for the $840-\mathrm{km}$ dispersion. This is in contrast to the minimum of ten pilot tones using an equal spacing design with either cubic or Fourier-transform-based interpolation.
\end{abstract}

Keywords: Optical fast OFDM, dispersion, coherent detection

\section{Introduction:}

Optical orthogonal frequency division multiplexing (OFDM) [1-7] has drawn much interest recently for long-haul transmission systems [1,2] as well as access networks [3,4] due to its enhanced spectral efficiency and high dispersion tolerance. One of major concerns in the implementation of optical OFDM is the channel estimation, which can be achieved by inserting training signals in the frequency domain (pilot tones), time domain (training symbols), or the combination of both. Clearly, the number of pilot tones and/or training symbols should be as small as possible to avoid the transmission overhead, and rapid estimation is needed for better acquisition and tracking of the channel response. This is of particular importance to burst-mode transceivers for optical packet networks where the signal is detected on a packet-by-packet basis. The optimal design of the pilot tones to keep the overhead at the minimum depends on the channel characteristics. In wireless communications, it was theoretically verified that in additivewhite-Gaussian-noise based channel, the pilot tones should be equally spaced to maximize the signal-to-noise ratio [5]. In direct-detected optical OFDM [7], it was shown that the design with pilot tones spaced more densely at lower frequencies where the optical signal to noise ratio was higher could result in better estimation performance. However, as shown in this paper, this result cannot be applied to coherent detection in the presence of chromatic dispersion (CD).

Optical fast OFDM (F-OFDM) is a promising OFDM scheme [8-12], where the subcarrier spacing is reduced to the half of that in conventional OFDM. This scheme exhibits significantly improved performance in frequency offset compensation when compared to conventional OFDM [9], so is more suitable for fast tunable transceivers. The subcarrier multiplexing/demultiplexing can be implemented by using a discrete cosine transform (DCT) pair. Due to the different properties of optical F-OFDM and conventional optical OFDM, a symmetric extension rather than cyclic extension based guard interval (GI) is required in optical F-OFDM to enable CD compensation using one-tap equalizers.

In this paper, we investigate the optimal design of pilot tones in CD-limited coherent optical F-OFDM. We theoretically show that inserting pilot tones with frequency intervals inversely proportional to the subcarrier index minimizes the overhead. With the proposed design, a 20- 
Gbit/s four amplitude shift keying optical F-OFDM system with 840-km transmission without optical dispersion compensation is experimentally demonstrated. It is shown that this design exhibits greatly improved performance when compared to the equal spacing design, and a single F-OFDM symbol with six pilot tones can achieve near-optimal estimation performance for 840$\mathrm{km}$ dispersion. The presented concept can also be potentially applied to conventional coherent optical OFDM.

\section{Principle}
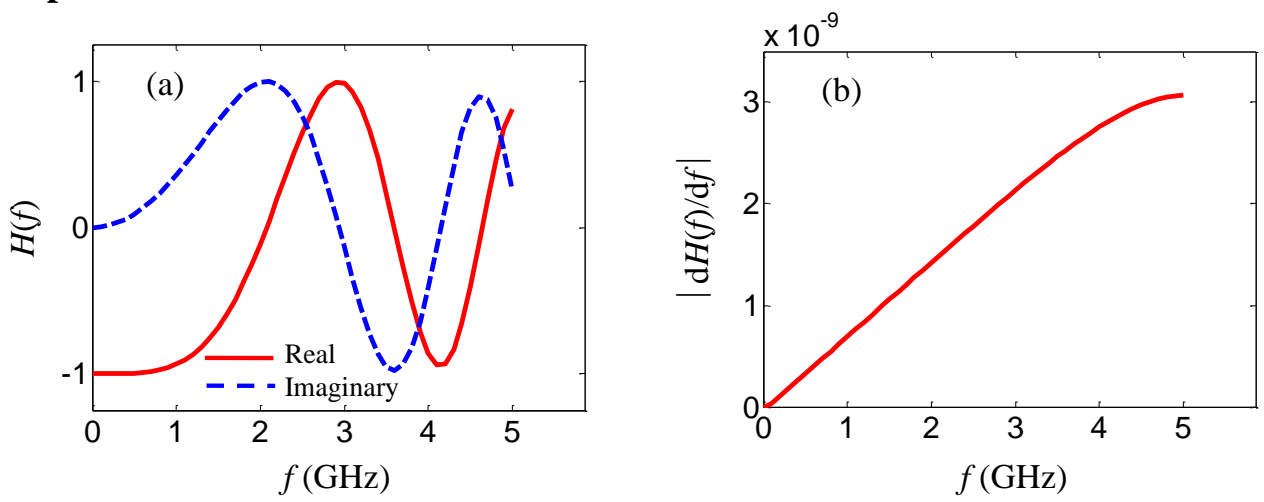

Fig. 1. An example of (a) $H(f)$ and (b) $|\mathrm{d} H(f) / \mathrm{d} f|$ as a function of $f$.

In a coherent transmission system in the presence of $\mathrm{CD}$, the channel response, $H(f)$, can be written as:

$$
H(f)=A \cdot H_{s}(f) \cdot \exp \left(j \cdot \beta_{2} \cdot L \cdot(2 \pi f)^{2} / 2\right)
$$

where $A$ is the channel gain/loss, $H_{s}(f)$ represents the static response including the contributions of electrical driver, modulator, and receiver, and $\beta_{2} L$ is the accumulated $\mathrm{CD}$ value. The goal of the proposed scheme is to reconstruct $H(f)$ with the minimum number of pilot tones. Fig. 1(a) shows an example of $H(f)$ as a function of $f$. In the figure, $H_{s}(f)$ is assumed as a 3-order Gaussian-shaped low-pass filter with 3-dB bandwidth of $6 \mathrm{GHz} . A=-1, \beta_{2}=-22 \mathrm{ps}^{2} / \mathrm{km}$, and $L=840 \mathrm{~km}$. From the figure, it can be seen that the channel response oscillates rapidly for high frequencies. In fact, the oscillation frequency of $H(f), \mathrm{d} H(f) / \mathrm{d} f$, is derived as:

$$
\begin{aligned}
& \frac{d H(f)}{d f}=A \cdot \exp \left(j \cdot \beta_{2} \cdot L \cdot(2 \pi f)^{2} / 2\right) \cdot \frac{d H_{s}(f)}{d f} \\
&+A \cdot H_{s}(f) \cdot j \cdot \beta_{2} \cdot L \cdot\left(4 \pi^{2} f\right) \cdot \exp \left(j \cdot \beta_{2} \cdot L \cdot(2 \pi f)^{2} / 2\right)
\end{aligned}
$$

Note that in practice, $H_{s}(f)$ is close to a constant, especially when pre- and/or post-equalization is applied in the digital domain. Therefore, in CD-limited systems, $\mathrm{d} H(f) / \mathrm{d} f$ is dominated by the second term on the right-hand side of Eq. (2). Fig. 1(b) illustrates $|\mathrm{d} H(f) / \mathrm{d} f|$ where the parameters of $H(f)$ are the same as those in Fig. 1(a). The figure clearly indicates that $|\mathrm{d} H(f) / \mathrm{d} f|$ is approximately linearly proportional to $f$. Based on the sampling theorem, pilot tones should be inserted more frequently for larger $f$ values for better reconstruction of the frequency response. Specifically, the pilot tone density should increase linearly with $f$. In the discrete domain, the frequency interval between pilot tones is inversely proportional to the subcarrier index. Assuming that $M$ and $N$ are the numbers of total subcarriers and pilot tones, respectively, the frequency intervals between pilot tones are designed to be approximately $1: 1 / 2: 1 / 3 \ldots: 1 / N$, and the position of the $i^{\text {th }}$ pilot tone, $P_{\mathrm{i}}$, is designed as:

$$
P_{i}=\operatorname{ceil}[(1+1 / 2+\ldots+1 /(i-1)) \times M /(1+1 / 2 \ldots+1 / N)+1] \quad i \geq 2
$$


ceil[.] rounds the value to the nearest integer towards infinity. In this paper, the full channel response is obtained using cubic-based interpolation. For comparison, equally spaced pilot tones are also investigated where the frequency intervals between pilot tones are approximately the same $(=M / N)$ and the position of the $i^{\text {th }}$ pilot tone, $P_{\mathrm{i}}$, is:

$$
P_{i}=\operatorname{ceil}[(i-1) \times M / N+1] \quad i \geq 2
$$

The full channel response is reconstructed using either cubic or discrete Fourier transform (DFT) based interpolation in this scenario, for comparison with the proposed design. Given the same $M$ and $N$, the computation complexity of cubic based interpolation is similar for non-uniformly and uniformly spaced pilot tones. However, the DFT-based interpolation is commonly applied to equally spaced pilot tones.

\section{Experimental setup}

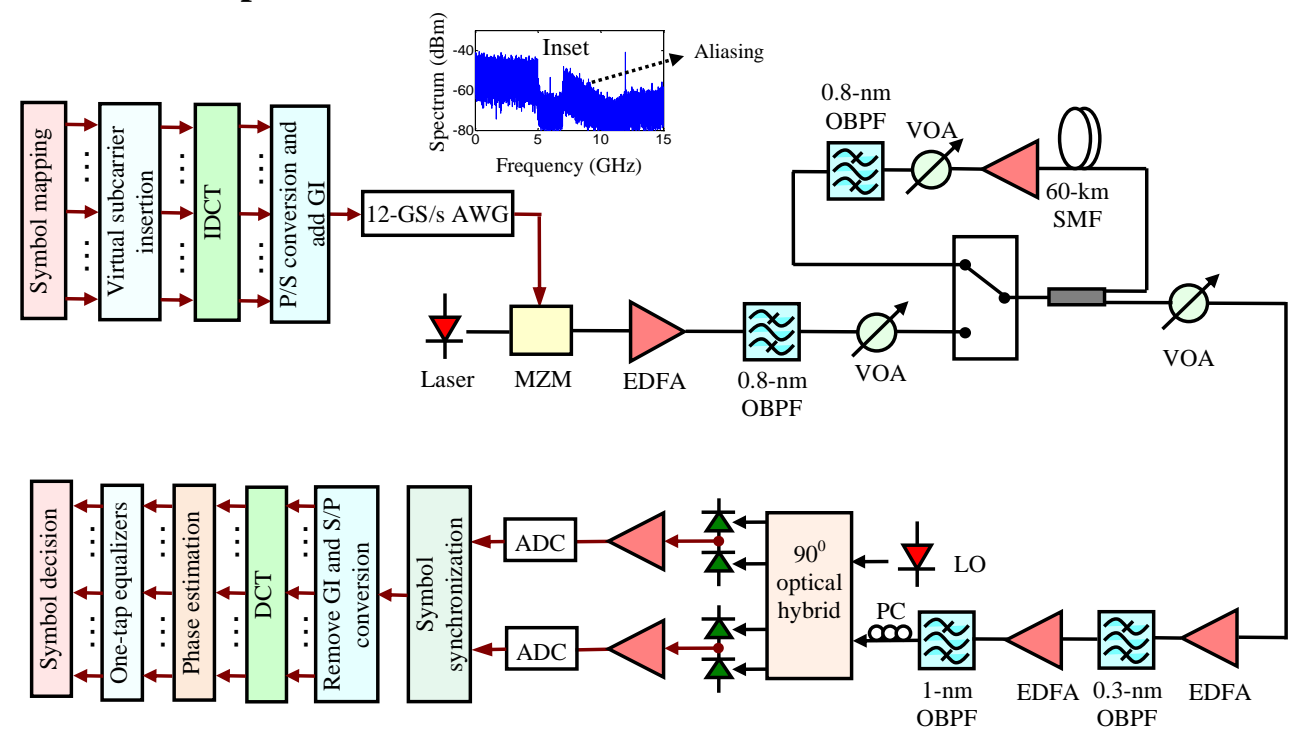

Fig. 2. Experimental setup of coherent optical F-OFDM.

Fig. 2 shows the experimental setup. The four amplitude shift keying (4-ASK) F-OFDM signal was encoded with Gray code in Matlab. The IDCT used 256 points, of which 213 subcarriers (\#2\#214) were used for data transmission. The first subcarrier was not modulated, allowing for ACcoupled drive amplifiers and receivers. The last 42 subcarriers were zero-padded to avoid aliasing. After IDCT and parallel-to-serial (P/S) conversion, 12 samples were added to each symbol as a symmetric extension based GI. The signal was pre-equalized to compensate the bandwidth limitation of the arbitrary waveform generator (AWG) and clipped to maintain 11-dB peak-to-average-power ratio. The generated signal was uploaded into a 12-GS/s AWG. The bandwidth of the AWG excluding the sinc-function roll off was around 7.5 GHz. The nominal signal data rate including the GI and forward error correction was $\sim 20 \mathrm{Gbit} / \mathrm{s}(213 / 256 \times 6 \times 4)$. The inset of Fig. 1 depicts the electrical spectrum after the AWG, where the electrical bandwidth for the 20-Gbit/s 4-ASK F-OFDM signal was $5 \mathrm{GHz}$ and the frequency components beyond $7 \mathrm{GHz}$ were due to the aliasing. A fibre laser with $6-\mathrm{kHz}$ linewidth was used to generate the optical carrier. A Mach-Zehnder modulator (MZM) was used for signal modulation with a peak-to-peak signal input voltage of $0.5 V_{\pi}$. The modulated optical signal was then amplified by an erbium doped fibre amplifier (EDFA), filtered by a $0.8-\mathrm{nm}$ optical band-pass filter (OBPF), and transmitted over a recirculating loop comprising $60-\mathrm{km}$ single-mode fibre (SMF) with $\sim 14 \mathrm{~dB}$ of fibre loss. The noise figure of the loop EDFA was $5 \mathrm{~dB}$ and another 0.8-nm OBPF was used in 
the loop to suppress the amplified spontaneous emission noise. The launch power per span was $4.5 \mathrm{dBm}$. At the receiver, the optical signal was detected using a pre-amplified coherent receiver and a variable optical attenuator (VOA) was used to control the optical signal-to-noise ratio (OSNR). The pre-amplifier was followed by an OBPF with a $3-\mathrm{dB}$ bandwidth of $0.3 \mathrm{~nm}$, a second EDFA, and another optical filter with a 3 -dB bandwidth of $1 \mathrm{~nm}$. A polarization controller (PC) was used to align the polarization of the filtered F-OFDM signal before it entered the signal path of a $90^{\circ}$ optical hybrid. The outputs of the hybrid were connected to two balanced photodiodes with $40-\mathrm{GHz} 3-\mathrm{dB}$ bandwidths, amplified, and captured using $16-\mathrm{GHz}$ 50-GS/s analogue-todigital converters. The decoding algorithms included symbol synchronization, DCT, phase estimation, and one-tap equalizers to compensate the CD. The coefficients of the one-tap equalizers were estimated using pilot tones with subsequent interpolation. Due to the pre-filtering at the transmitter and post-equalization at the receiver, the signal-to-noise ratio loss at higher signal frequencies was not significant. Eight hundred F-OFDM symbols were measured, giving a total number of measured 4 -ASK data of $213 \times 800=170,400$. The bit error rate (BER) was obtained using error counting with optimal decision thresholds for each subcarrier data. Fig. 3(a) shows the real and imaginary tributaries of the experimentally obtained channel response after $840 \mathrm{~km}$, and as theoretically predicted in Fig. 1(a), the curves oscillate more rapidly for larger subcarrier index. The channel response can be represented by a group of orthogonal functions with their coefficients being the DFT of the response, as depicted in Fig. 3(b). In theory, the number of non-zero coefficients is the minimal number of required pilot tones to reconstruct the response [5], so Fig. 3(b) can provide a guideline on how close different designs approach the theoretical limit. In practice, most of the coefficients may not be zero, as can be seen in Fig. 3(b). However, the channel response can be well estimated by $5 \sim 6$ dominant coefficients.
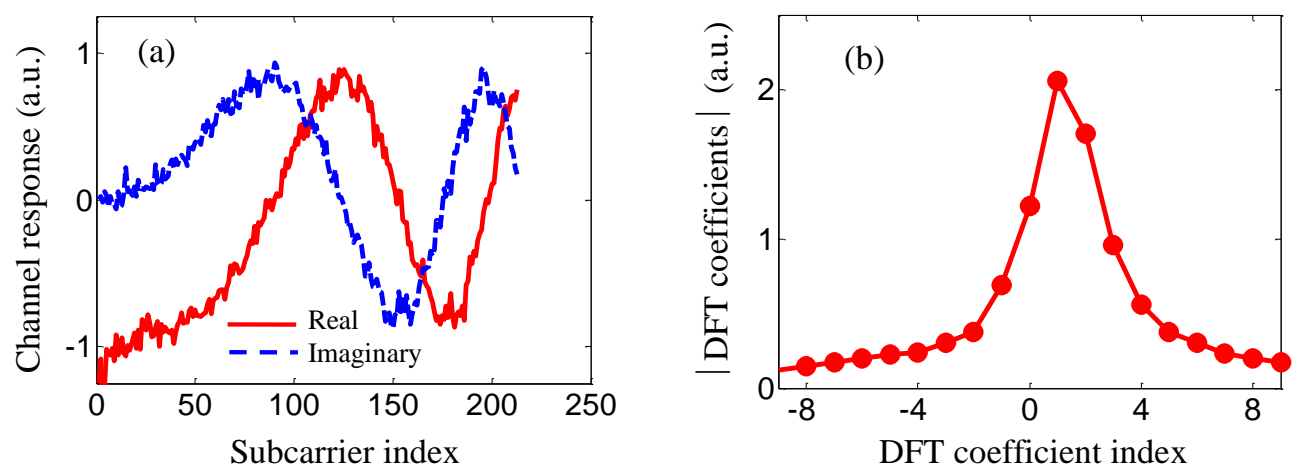

Fig. 3. (a) Real and imaginary tributaries of the channel response obtained from the experiment after 840 $\mathrm{km}$ and (b) its DFT coefficients.

\section{Experimental results}

Fig. 4 shows BER versus OSNR at $840 \mathrm{~km}$. The proposed pilot tone design was compared with the equal spacing design with (a) cubic- and (b) DFT-based interpolation. Circles in (a) represent the reference BER obtained from full channel estimation (all subcarriers as pilot tones). The number of optical F-OFDM symbols for channel estimation was 20, i.e. the channel response obtained at the pilot-tone frequencies was averaged over 20 symbols before interpolation. The figures show that for the equal spacing design, cubic and DFT based interpolation may result in different performance. However in both cases, BER was around $10^{-2}$ at $17.1-\mathrm{dB}$ OSNR when the number of pilot tones was six. Increasing the pilot tone number improved the estimation performance, and only ten pilot tones could obtain similar BER as that using full channel estimation. In contrast, by using the proposed method, six pilot tones could achieve near-optimum estimation performance with BER of $3.3 \times 10^{-4}$ at 17.1-dB OSNR, representing more than one order of magnitude BER improvement when compared to the equal spacing design with the same 
pilot tone number. Note that from the discussion in section 3, six is close to the minimal required value for $840 \mathrm{~km}$ because from Fig. 3(b), the channel response has to be approximated by at least $5 \sim 6$ dominant coefficients. Also note that the electrical bandwidth of the signal in the experiment was $5 \mathrm{GHz}$, and the benefit of the proposed method would be much more significant for higher symbol rate with the use of higher-speed digital-to-analogue converters such as $56 \mathrm{GS} / \mathrm{s}$.
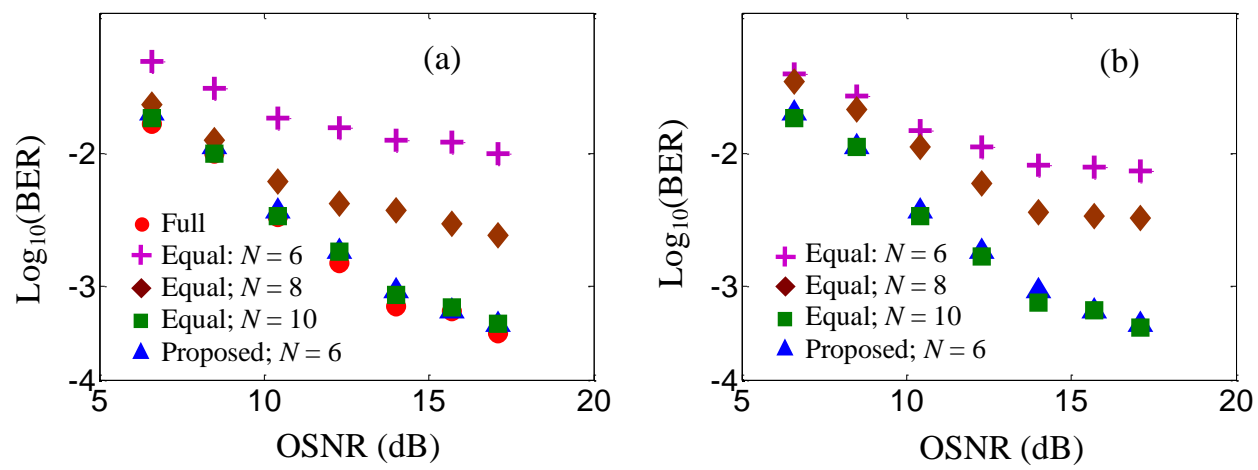

Fig. 4. BER versus OSNR at $840 \mathrm{~km}$. The proposed design is compared with the equal spacing design with (a) cubic- and (b) DFT-based interpolation.

Fig. 5 depicts BER versus the number of optical F-OFDM symbols for channel estimation using the proposed design and the equal spacing design with (a) cubic- and (b) DFT-based interpolation at $840 \mathrm{~km}$. The OSNR is $17.1 \mathrm{~dB}$. It is confirmed that the proposed design could achieve the optimal performance with a smaller number of pilot tones (six), while similar performance could only be obtained by ten pilot tones in the equal spacing design. Note that in Fig. 5(b), the equal spacing design with ten pilot tones resulted in slightly better performance than the proposed design with six pilot tones, which however was negligible. It is also seen that in the experiment, a single F-OFDM symbol could enable correct dispersion estimation with the obtained BER below $1 \times 10^{-3}$. Note that although $840 \mathrm{~km}$ was investigated in Fig. 5, six pilot tones are sufficient for any distance below $840 \mathrm{~km}$. Therefore, optical F-OFDM using the proposed design can track the channel rapidly for a distance range of up to $840 \mathrm{~km}$ at the minimum overhead, so is potential for burst-mode applications in optical packet networks.
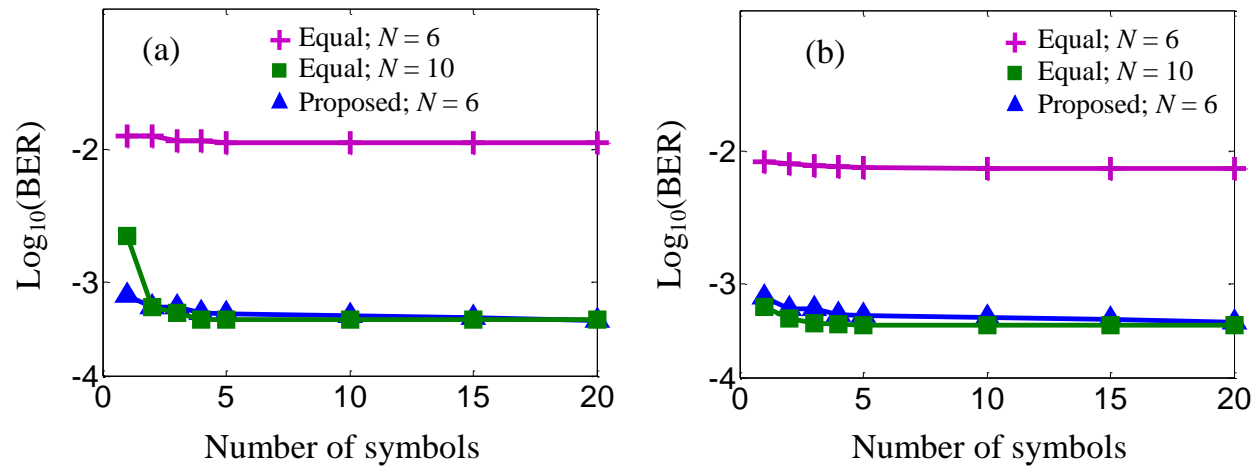

Fig. 5. BER versus the number of F-OFDM symbols used for channel estimation.

\section{Conclusions}

We have investigated the optimal design of pilot tones for channel estimation in dispersionlimited coherent optical F-OFDM systems. It is shown, in a 20-Gbit/s 4-ASK optical F-OFDM experiment with $840-\mathrm{km}$ transmission without optical dispersion compensation, that by inserting 
pilot tones with frequency interval inversely proportional to the subcarrier index, a single optical F-OFDM symbol with six pilot tones can achieve near-optimal estimation performance. This is in contrast to the minimum of ten pilot tones using the equal spacing design with either cubic or DFT-based interpolation. The proposed design makes optical F-OFDM an attractive solution for spectrally efficient optical packet networks.

\section{Acknowledgements:}

This work was supported by Science Foundation Ireland under grant number 11/SIRG/I2124 and 06/IN/I969, and the EU $7^{\text {th }}$ Framework Program under grant agreement 318415 (FOX-C).

\section{References:}

[1] W. Shieh and C. Athaudage, Electron. Lett. 42 (2006) 587-589.

[2] A.J. Lowery and J. Armstrong, Opt. Express 14 (2006) 2079-2084.

[3] C.H. Yeh, C.W. Chow, H.Y. Chen, and B.W. Chen, Opt. Express 19 (2011) 26150-26160.

[4] J. Xu, A. Ali, C. Ruprecht, J. von Hoyningen-Huene, J.N. Zhao, J. Leibrich, and W. Rosenkranz, in Proc. Optical Fiber Communication Conference (2012) paper OW4B.

[5] R. Negi and J. Cioffi, IEEE Transactions on Consumer Electronics 44, (1998) 1122-1128.

[6] L. Liu, X. Yang, and W. Hu, in Proc. Asia Communications and Photonics Conference (2011), paper 830937.1-6.

[7] F. Li, Z. Cao, G. Wen, J. Xiao, J. Yu, and L. Chen, Optics Communications 285 (2012) 3075-3081.

[8] S.K. Ibrahim, J. Zhao, D. Rafique, J. O'Dowd, and A.D. Ellis, in Proc. European Conference on Optical Communication (2010), post-deadline paper PDP3.4.

[9] J. Zhao and A.D. Ellis, IEEE Photon. Technol. Lett. 24 (2012) 2284-2287.

[10] J. Zhao, S.K. Ibrahim, D. Rafique, P. Gunning, and A.D. Ellis, IEEE Photon. Technol. Lett. 23 (2011) 594-596.

[11] W. Long, J. Zhang, D. Wang, J. Han, S. Chen, A.M. Han, G. Gao, H. Leng, L. Liu, W. Zhu, Y. Zhao, and W. Gu, IEEE Photon. Technol. Lett. 24 (2012) 1160-1162.

[12] E. Giacoumidis, I. Tomkos, and J.M. Tang, in Proc. Optical Fiber Communication Conference (2011) paper OWU3. 